\title{
A Novel In Vitro Model of Trophoblast-Mediated Decidual Blood Vessel Remodeling
}

\author{
Caroline Dunk, Ljiljana Petkovic, Dora Baczyk, Janet Rossant, Elke Winterhager, \\ and Stephen Lye
}

\begin{abstract}
Departments of Physiology and Fetal and Maternal Health (CD, LP, DB, JR, SL), Samuel Lunenfeld Research Institute, Mount Sinai Hospital, Toronto, Ontario, Canada; and Institute of Anatomy (EW), University of Essen,
\end{abstract} Essen, Germany

\begin{abstract}
SUMMARY: In vivo the extravillous trophoblasts (EVTs) penetrate the decidua and the first third of the myometrium to remodel the uterine spiral arteries and achieve the high-flow, low-resistance circulation characteristic of the intervillous space of the term placenta. Much of our understanding of these processes comes from histologic analysis of placental bed biopsies, a limited tissue source and one that can provide only a snapshot of a dynamic process. To better characterize these cellular interactions, we have developed an in vitro co-culture system in which first trimester villous explants are cultured at low oxygen tension in contact with $2-\mathrm{mm}^{2}$ sections of decidua parietalis from the same patient. Hematoxylin eosin counterstaining of paraffin sections shows that EVT columns form at the tips of the placental villi and adhere and penetrate the decidual surface. The decidual blood vessels in the path of the EVT show morphologic disruption. Immunohistochemical analysis of the co-cultures using both an endothelial specific anti-CD31 and an anti-smooth muscle actin antibody show a disruption of the integrity of the vessel lining together with a complete loss of organized smooth muscle actin surrounding the blood vessels. In contrast control decidua samples in the absence of placental villi exhibit blood vessels with a complete endothelial lining and an organized muscular sheath. Using both an anti-cytokeratin-7 and anti-Cdx-2 antibody specific to trophoblasts, we show that these changes coincide with invasion of the vessels by endovascular trophoblasts and penetration of the decidua by interstitial EVTs. No EVTs were found in the control decidua. Thus we conclude that this in vitro model mimics the physiologic change observed in vivo during trophoblast invasion into maternal decidual tissues, and as such it may provide useful information concerning the interactions between EVTs and decidual cells and vessels during early gestation. (Lab Invest 2003, 83:1821-1828).
\end{abstract}

$I$ $\mathrm{n}$ normal human pregnancy, the blastocyst attaches to the uterine epithelium and implants into the endometrial stroma, giving rise to the extraembryonic trophoblast shell, the precursor of the hemochorial placenta. Simultaneously the endometrial stroma transforms into the decidua under the influence of progesterone. Decidualization is characterized by the enlargement of endometrial stromal cells, the growth of the uterine spiral arteries, and infiltration of the stroma by macrophage and large granular lymphocytes or uterine natural killer cells (Benirschke and Kaufmann, 1995). In hemochorial placentation the trophoblasts differentiate along two main pathways: villous and extravillous. The villous trophoblast comprises the cytotrophoblast and the overlying syncytiotrophoblast of the villous tree, which is bathed by maternal blood in the intervillous space. Extravillous trophoblasts (EVTs) are derived from the tips of some anchoring villi where villous cytotrophoblast cells pro-

\section{DOI: 10.1097/01.LAB.0000101730.69754.5A}

Received August 17, 2003.

This work was supported by a grant from the NIH (HD42558-01) and by a fellowship from the Canadian Institutes of Health Research (to CD).

Address reprint requests to: Dr. S.J. Lye, Samuel Lunenfeld Research Institute, Mount Sinai Hospital, Room 982, 600 University Avenue,

Toronto, Ontario, Canada, M5G1X5.E-mail: lye@mshri.on.ca liferate to form the cell columns from which individual EVTs separate, invade, and migrate into the maternal decidua. (Aplin, 1991; Loke and King, 1995).

The first trimester of trophoblast invasion and placental development occurs in a low-oxygen environment, as the blood flow to the intervillous space is not yet established (Genbacev et al, 1996; Rodesch et al, 1992). During this time the anchoring villi of the developing placenta give rise to two populations of EVT: the interstitial (InEVT) and the endovascular (EnEVT). The InEVTs invade as far as the superficial layer of the myometrium and appear to preferentially home toward the uterine spiral arteries and encircle them (Pijnenborg et al, 1980, 1983). The EnEVTs arise as groups of trophoblast cells, which detach from the trophoblastic shell and invade the lumen of the decidual uterine spiral arteries. The EnEVTs form loose plugs in the apical portions of these vessels, and communication with the intervillous space is restricted to a network of intercellular channels (Burton et al, 1999). Concomitant with trophoblast invasion, the walls of the maternal spiral arteries are destroyed. Fibrinoid necrosis of the vessel media and a loss of endothelium occurs, converting muscular vessels into flaccid sinusoidal sacs. (Boyd and Hamilton, 1970). This vascular transformation is necessary to accommodate the huge increase in blood flow (3.5-fold) to the intervillous space required to successfully support 
the developing fetus. (Thaler et al, 1990; Ziegler et al, 1999).

There is some controversy as to the roles of the two EVT populations in the transformation of the spiral arteries. Immunohistochemical studies of placental bed biopsies suggest that EnEVT and endothelial cells transiently coexist on the walls of partially modified spiral arteries. The EnEVT are thought to migrate along the vessel lumen and adopt a more endothelial cell phenotype as they replace the endothelium and most of the musculoelastic tissues of the vessel walls (Pijnenborg et al, 1980; Zhou et al, 1997b). However, a study examining the change in decidual spiral arteries found that medial necrosis and replacement with fibrinoid material only occurred in the presence of InEVTs, whereas it was the EnEVTs that were responsible for replacing the endothelial cells in the transformed arteries (Kam et al, 1999). These authors suggest that the InEVTs prime the vessels in some way for invasion by the EnEVTs (Kam et al, 1999). Failure of the EnEVTs to acquire an endothelial phenotype and to proceed beyond the superficial portions of the spiral arteries is thought to contribute to complications of pregnancy such as intrauterine growth retardation, pre-eclampsia, and spontaneous miscarriage (Lim et al, 1997; Lyall, 2002; Zhou et al, 1997a). Previous work has shown that the interstitial invasion is not affected in these cases (Pijnenborg et al, 1998), suggesting that the ability of the EnEVTs to enter and interact with the vascular cells of the spiral arteries may be the key difference between normal and preeclampsia and intrauterine growth retardation pregnancies (Khong et al, 1986). However, more recent studies have reported a decrease in the depth of interstitial invasion in pre-eclamptic pregnancies (Kadyrov et al, 2003), which in turn may impact the endovascular invasion. These conflicting reports highlight the need for further understanding of the mechanisms behind these critical cell-to-cell interactions in both normal and pathologic pregnancies.

Studies of trophoblasts and spiral artery interactions have been confined to immunohistochemical analysis of placental bed biopsies and pregnant hysterectomies (Kam et al, 1999; Lyall et al, 1999, 2000; Zhou et al, 1997a, 1997b), which are limited to a single time point. In vitro studies have also been limited by a lack of suitable models, although recently a study investigating the perfusion of isolated myometrial arteries with either primary trophoblast or EVT cell lines has shown that both interstitial and endovascular interactions could be detected (Cartwright et al, 2002). However, these authors used myometrial tissues from term pregnancies, which may not be relevant to the cellular interactions that occur during the first trimester within the decidua. In this study we present a novel in vitro model of decidual spiral artery remodeling using co-cultures of first trimester decidua parietalis and placental explants.

\section{Results and Discussion}

First trimester placental villi cultured in contact with the apical epithelial surface of the decidua adhere to the decidual fragments (Fig. 1A) and show the forma- tion of EVT cell columns that penetrate the decidua in paraffin sections (Fig. 1B). Immunohistochemical analysis of these co-culture sections using a monoclonal anti-cytokeratin-7 (CK-7) antibody demonstrated strong immunoreactivity in the villous trophoblasts of placental villi and the EVT cell column (Fig. 1C). Moreover, both InEVTs and EnEVTs were detected within the decidual stroma and spiral arteries, respectively, extending from the trophoblast cell column into the decidua (Fig. 1C) over the culture period of 6 days. The EVTs appeared to preferentially enter the lumen of the arterial blood vessels where they formed loose cohesive structures in the proximal portions of the decidual spiral arteries, similar to the trophoblast plugs observed in vivo during early pregnancy (Fig. 1D). Beyond the trophoblastic plugs, the endovascular trophoblasts appeared to migrate down the endothelial surface, causing a disruption of the endothelial cell integrity as they moved and relined the vessel with EnEVT (Fig. 1, D and F). The EnEVTs also appeared to invade the vessel wall, enter the decidual stroma, and migrate toward other blood vessels becoming InEVTs (Fig. 1C). In this model the uterine veins are never invaded, showing no staining with $\mathrm{CK}-7$ and an intact endothelium (Fig. 1, D and E; Fig. 2, A to D). In a total of 29 vessels ( $n=$ three to six per experiment) analyzed from six experiments, invasion by trophoblasts occurred in $69.86 \pm 6.1 \%$ in the proximal regions of the decidua and extended to the distal portions in $53.2 \% \pm 7.2 \%$ by 6 days of culture. Study of earlier time points revealed that EVT columns required 3 days to attach the placenta to the decidua; and by 4 days of culture, the proximal arteries displayed signs of invasion, which extended to the distal portions by the 6th day.

In the present study, CK-7 staining was also routinely detected in the epithelium of decidual glands of both invaded and noninvaded tissues (Fig. 1G). It should be noted that longitudinal sections of decidual glands distinctly resemble transformed spiral arteries displaying a layer of CK-7 positive cells, a lack of endothelium and small focal depositions of smooth muscle actin (SMA). Therefore, great care should be taken in the identification and analysis of decidual blood vessel remodeling when using CK-7 as a marker to identify invaded trophoblasts. In this study we have used a monoclonal antihuman caudal-related homeobox gene-2 (Cdx-2) antibody as a potentially more specific marker of trophoblasts in the placentaldecidual co-cultures. Cdx-2 has previously been identified as an intestine-specific transcription factor where it is thought to regulate the proliferation and differentiation of intestinal epithelial cells during crypt formation (Silberg et al, 2000). Beck et al (1995) reported the early expression of $\mathrm{Cdx}-2$ in the trophectoderm of Day 3.5 mouse embryos and continuing expression in the fetal membranes and spongiotrophoblasts at later stages, suggesting that this homeobox gene may also be involved in placental development. In this study the placental-decidual cocultures displayed strong Cdx-2 immunostaining in the EVT cell column (Fig. 1J) and in EnEVT lining 

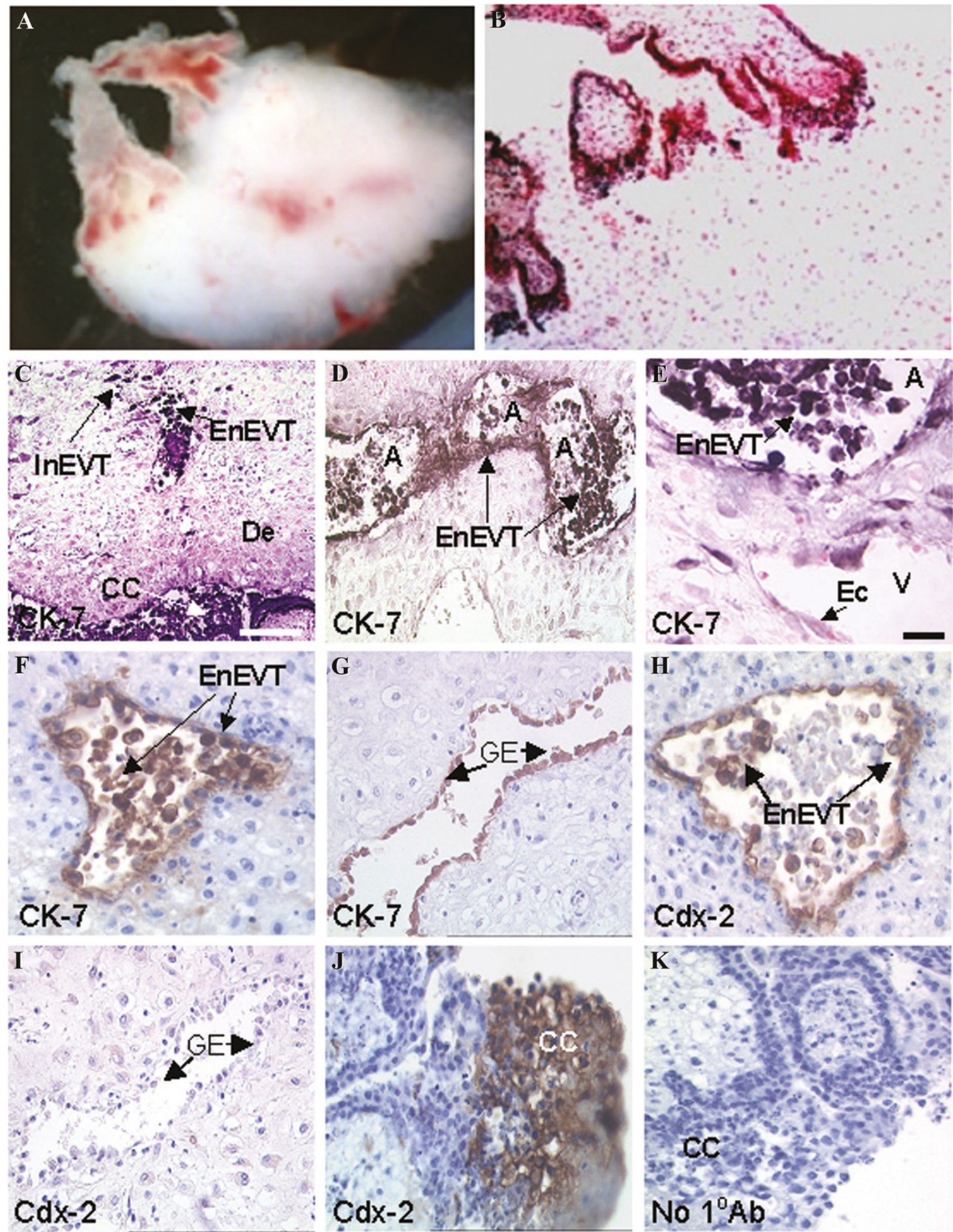

No $1^{\circ} \mathrm{Ab} \div$

\section{Figure 1.}

(A) Photomicrograph of a placental-decidual co-culture (8 weeks gestational age). (B) Hematoxylin eosin counterstaining of a cross-section of placental-decidual co-culture showing extravillous trophoblast (EVT) cell columns penetrating the decidua. All sections shown are from Day 6 of culture. (C) Cytokeratin (CK)-7 immunostaining in the trophoblast cell column (CC), interstitial EVT (InEVT) within the decidual stroma (Dec), and endovascular EVT (EnEVT) within the spiral arteries $(A)$. (D to E) CK-7 immunostaining in EnEVTs within the spiral artery trophoblast plugs are visible, and the EnEVTs have replaced the endothelium. (E) The uterine veins $(V$ are not invaded. $(F)$ Invaded decidual spiral artery stained with anti-CK-7 antibody to identify trophoblast. (G) CK-7 was routinely detected in the glandular epithelium (GE) of decidual glands. $(\mathrm{H})$ In serial sections $\mathrm{Cdx}-2$ immunostaining was also detected in the EnEVTs in the vessel lumen and lining the transformed arteries of the placental-decidual co-culture. (I) Cdx-2 antibody does not stain glandular epithelium in serial sections. (J) Cdx-2 antibody stained positively in the distal invasive trophoblast of the cell column. (K) Negative control: omission of the primary antibody. Scale: $\mathrm{C}$ and $\mathrm{D}$ bars represent $50 \mu \mathrm{m}$; $\mathrm{E}$ to $\mathrm{K}$ bars represent $25 \mu \mathrm{m}$. 

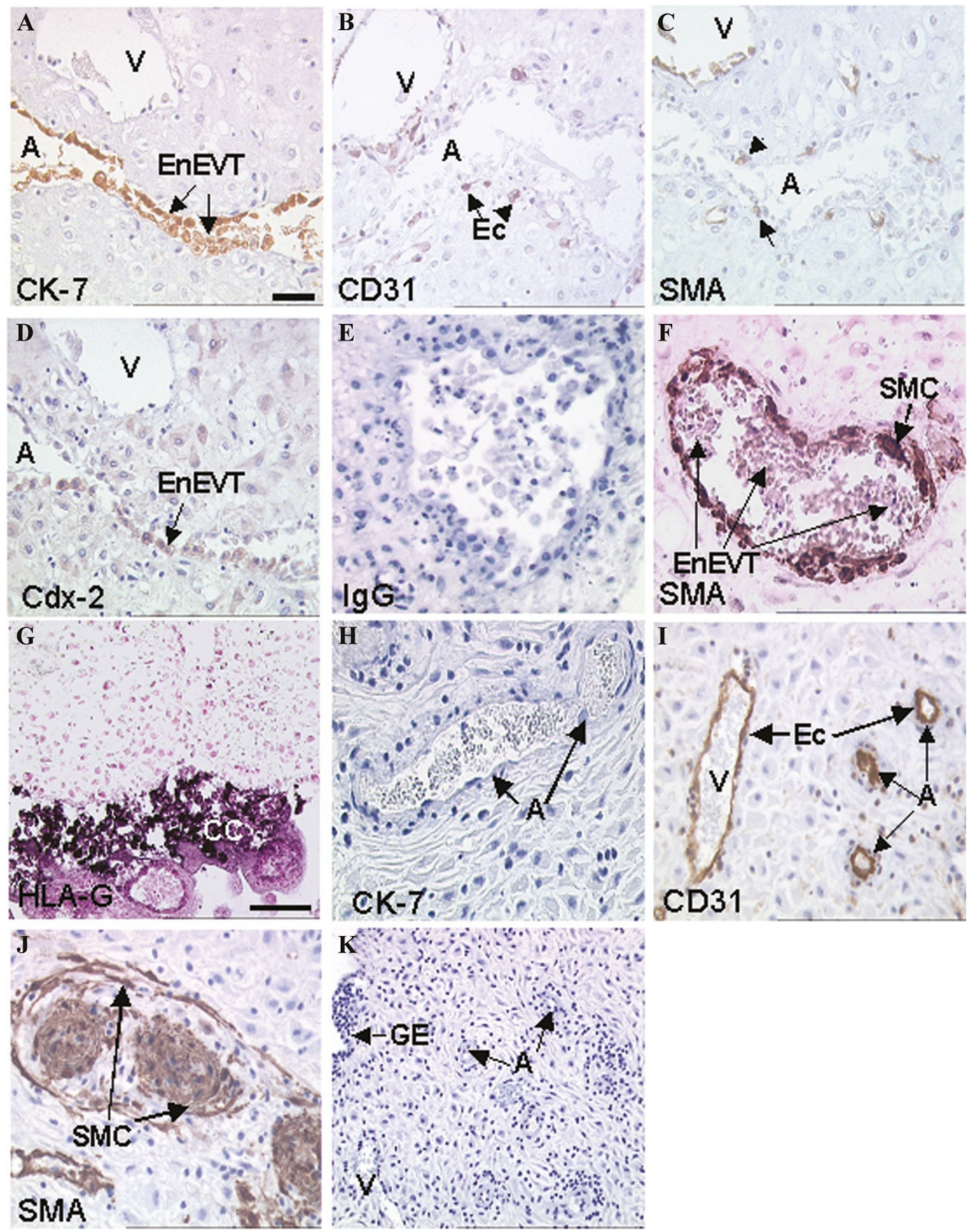

Figure 2.

All sections shown are from Day 6 of culture. (A to E) Serial sections. (A) Some arteries were observed in which the endovascular extravillous trophoblast (EnEVT) had completely replaced the endothelium (cytokeratin [CK-7]). (B) CD31 antibody stained residual isolated endothelial cells (EC) underlying EnEVT. (C) Smooth muscle actin (SMA) antibody stained small focal areas of actin underlying the remaining endothelial cells; all outer layers of actin were completely lost. (D) Cdx-2 immunoreactivity localized to EnEVTs relining the vessels. (E) Negative control: nonimmune mouse IgG. (F) SMA. Smooth muscle cells (SMCS) remain in the partially invaded vessels in areas not contacted by EnEVT. (G) Soluble human leukocyte antigen (HLA)-G was detected in the EVT cell column (cc) only (diaminobenzidine + nickel and nuclear fast red counterstain). (H to K) Control decidual parietalis. (H) No EnEVTs or interstitial EVTs were observed in the control decidua shown by immunostaining using anti-CK-7. (I) CD31. Intact endothelium was observed in the control decidua parietalis; arterioles $(A)$ demonstrated a swollen endothelium, while veins ( $V$ had a smooth flat endothelium. (J) SMA antibody detected complete rings of SMCs surrounding the blood vessels; the larger spiral arteries demonstrated many layers of smooth muscle and medial cells. (K) Negative control: omission of primary antibody. $G E=$ glandular epithelium. Scale: $\mathrm{A}$ to $\mathrm{F}$ and $\mathrm{H}$ to $\mathrm{J}$ bars represent $25 \mu \mathrm{m} ; \mathrm{G}$ and $\mathrm{K}$ bars represent $50 \mu \mathrm{m}$. 
decidual spiral arteries (Fig. $1 \mathrm{H}$ ). This is the first identification of $\mathrm{Cdx}-2$ as a novel marker of human trophoblasts. In contrast, in both the control decidua parietalis and placental-decidual co-culture, no Cdx-2 immunostaining was detected in the glandular epithelium, decidual stroma, or endothelial cells (Fig. 1I). Comparison of the CK-7 and Cdx-2 immunostaining in adjacent serial sections has allowed us to identify and distinguish between true transformed blood vessels lined with EnEVTs (CK-7, Fig. 1F; Cdx-2, Fig. 1H) and epithelial glands (CK-7, Fig. 1G; Cdx-2, Fig. 1I). Interestingly, in mouse placental development, the spongiotrophoblasts can differentiate into giant cells, and in this way they are analogous to the cells of the cytotrophoblast cell columns that anchor the villi of the human placenta (Rossant and Cross, 2001). Therefore, we suggest that Cdx-2 may also be important in the differentiation of human EVTs. Interestingly Cdx-2 staining was not observed in all luminal EnEVTs (Fig. 2D) as compared with CK-7 (Fig. 2A). We hypothesize that this localization to a subset of EnEVT suggests a potential role for $\mathrm{Cdx}-2$ as a transcription factor in the differentiation of these invasive trophoblasts as they reline the blood vessels and interact with endothelium. The Cdx-2 staining therefore may reflect the different differentiated state of the EnEVT in an ongoing process of blood vessel transformation. These results identify Cdx-2 as a novel marker specific to the invasive trophoblasts of the human placenta.

Importantly, in the control decidua parietalis, no CK-7 or Cdx-2 immunostaining was detected in association with decidual blood vessels (Fig. 2G), showing that the EVTs observed in the placental decidual co-cultures were a result of de novo invasion in the experiment. In our model the trophoblast cells were observed to traverse the entire section of the decidua, migrating a distance of $5 \mathrm{~mm}$ over 6 days in culture. These results are in contrast to the observation made by Vicovac et al (1995), where in their placentaldecidual culture system, trophoblast invasion was limited to the superficial surface of the decidua. We suggest that this discrepancy may be accounted for by the fact that these authors cultured their first trimester explants at atmospheric oxygen tension, whereas our model system is cultured at $3 \% \mathrm{O}_{2}$ and $5 \% \mathrm{CO}_{2}$ (conditions equivalent to first trimester pregnancy). When our experiments were cultured at atmospheric oxygen tension, the placenta failed to firmly attach to the decidual surface and was easily disrupted by the fixation process (data not shown).

In the co-culture system, decidual spiral arteries were observed where the endothelium had been almost completely replaced by CK-7- and Cdx-2-positive EnEVTs (Fig. 2, A and D). To investigate the effect of this trophoblast infiltration on the decidual spiral arteries, we used both a monoclonal anti-CD31 (platelet endothelial cell adhesion molecule-1) and anti-SMA antibody in serial sections to identify endothelial and smooth muscle cells, respectively. In invaded spiral arteries, a loss of endothelium was observed with a few endothelial cells visible but disrupted and detached from the vessel wall. These vessels were lined by a mixed population of trophoblasts and isolated endothelial cells (Fig. 2B). Staining with the anti-SMA antibody revealed that in the arteries relined by trophoblasts, the muscle cells had been completely lost, and only flecks of SMA remained (Fig. 2C, arrowhead) underlying the remaining isolated endothelial cells. However, in the partially invaded spiral arteries, focal degradation of the smooth muscle was observed with a gradual loss of SMA staining from the periphery of the vessel and in areas where the luminal EnEVT plugs contacted the vessel wall (Fig. 2E). In contrast, in the control decidua parietalis, anti-CD31 detected a complete endothelial cell layer lining the spiral arterial blood vessels (Fig. 2H). Swelling of the endothelial cells, particularly in the smaller arteries, was apparent, as has been previously described in decidua parietalis (Kam et al, 1999). The veins also displayed an intact endothelial layer, but the cells showed no swelling appearing as a flat monolayer (Fig. 2H). In these tissues the anti-SMA antibody detected an intact ring of SMA-positive medial cells surrounding the smaller arteries, while the larger spiral arteries displayed many layers of prominent medial cells (Fig. 2l).

These results agree in part with former in vivo observations in which EnEVTs and endothelial cells are reported to transiently coexist on the walls of partially modified spiral arteries (Pijnenborg et al, 1980; Zhou et al, 1997b). However, in our study EnEVTs were often observed in decidual vessels that were not surrounded by InEVTs, in contrast with the in vivo reports by Kam et al (1999), in which EnEVTs were only observed in vessels surrounded by InEVTs. We suggest that this discrepancy may be explained by the artificial presentation of the placenta to the decidua in this model. In normal in vivo implantation, it is the trophectoderm and not the EVT that makes the initial contact with the uterine epithelium, and the invasive trophoblasts do not develop until the blastocyst is fully implanted into the uterine wall (Benirschke and Kaufmann, 1995). Therefore, we suggest that the later EVTs resulting from 7- to 9-week gestational placentae used in this study may not possess the full ability to attach to and penetrate the epithelium to give rise to InEVTs. However, in this model the EnEVTs efficiently enter the lumen of the decidual arteries, replacing the endothelium and degrading the smooth muscle, which suggests that the EnEVTs possess the ability to remodel decidual arteries independently with no requirement for interstitial priming. Recently there has also been some controversy surrounding the adoption of an endothelial phenotype by the EnEVTs as they contact and replace the endothelium. It has been reported that the EnEVTs mimic the adhesion phenotype of the endothelial cells that they replace and that EVTs in the cell columns, InEVTs, and EnEVTs express CD31 (Zhou et al, 1997b). In this study use of monoclonal antibodies specific to CK-7 and CD31 has shown that during invasion of the decidual blood vessels by the EnEVTs, there is no expression of CD31 in any of the trophoblast populations. The CD31 immunoreactivity observed localized only to maternal and fetal endothelial cells of intact vessels and to the 
remaining isolated endothelial cells in the invaded vessels. These observations are consistent with reports stating that there is no expression of CD31 in the EVTs of the placental bed in the first trimester of normal, pre-eclampsia, and intrauterine growth retardation pregnancies (Lyall et al, 2001; Pijnenborg et al, 1998).

The anti-human leukocyte antigen (HLA)-G has been reported to be a good marker of EVTs within the decidua (Hunt et al, 1991; Kovats et al, 1990). However, the HLA-G antibody used in the present study is specific to the soluble form of HLA-G (sHLA-G). Interestingly on the placental-decidual co-culture sections, this antibody detected antigen only in the distal part of the placental cell column (Fig. 2G) and not in the trophoblasts within the decidua that had stained positively for both CK-7 and Cdx-2 in serial sections (data not shown). This result agrees well with published work that reports strong expression of the sHLA-G at the fetal-maternal interface (Le Bouteiller et al, 1999), where it has been suggested to contribute to the elimination of potentially harmful maternal cytotoxic allogenic $\mathrm{T}$ cells during pregnancy (Le Bouteiller, 2000). This result suggests that the EVTs may stop secreting SHLA-G as they enter the decidua and that HLA-G expression may be restricted to the cellassociated membrane form found on InEVTs and EnEVTs in vivo (Proll et al, 1999).

In conclusion these results suggest that this model of first trimester trophoblast and decidual cell interactions is able to mirror the extensive changes that occur in vivo during the remodeling of the decidual spiral arteries during the formation of the utero-placental circulation. As such we suggest that this model provides an excellent opportunity for placentologists to investigate the mechanisms underlying the cellular interactions between fetal trophoblasts and maternal endothelium in both normal and pathologic pregnancies.

\section{Materials and Methods}

\section{Tissue Collection}

First trimester placentae and decidua parietalis were obtained at the time of elective terminations of pregnancy. Informed consent was obtained from each patient, and the University of Toronto review committee on the use of human subjects approved collections. Tissue from 6 to 9 weeks of gestation was dated according to the criteria of the Carnegie classification evaluating characteristics of embryonic/fetal parts. Placental tissue was washed in ice-cold PBS, and amnion and umbilical cord were dissected away. Decidua parietalis from the same patient was washed in ice-cold PBS and assessed for the integrity of the apical epithelial surface.

\section{Human Placental-Decidual Co-Culture}

Placental-decidual explant co-cultures were established from first trimester human tissues by a modification of the method of Vicovac et al (1995). Placental and decidual tissue of 6- to 9-weeks gestation was placed in ice-cold PBS and processed within 2 hours of collection. Small fragments of placental villi (15- to 20-mg wet weight) were dissected from the placenta, teased apart, and selected for the presence of trophoblast cell columns. Decidua parietalis was assessed for thickness and integrity of the sample, dissected to 2- to 3- $\mathrm{mm}^{2}$ cubes, and placed with the apical epithelial surface uppermost in Millicell-CM culture dish inserts (pore size $0.4 \mu \mathrm{m}$, Millipore Corporation, Bedford, Massachusetts) precoated with $0.2 \mathrm{ml}$ undiluted phenol red-free matrigel substrate (Becton Dickinson, Bedford, Massachusetts). The matrigel was allowed to solidify prior to the placement of the corresponding placental villous explant in contact with the decidual epithelial surface. Explants were cultured in 10\% FBS DMEM-Ham F-12 media (Life Technologies, Burlington, Ontario, Canada) supplemented with $100 \mu \mathrm{g} / \mathrm{ml}$ streptomycin, $100 \mathrm{U} / \mathrm{ml}$ penicillin, and $0.25 \mu \mathrm{g} / \mathrm{ml}$ ascorbic acid, $\mathrm{pH} 7.4$ at $3 \% \mathrm{O}_{2}$ and $5 \% \mathrm{CO}_{2}$. Culture medium was changed every 48 hours. Placentaldecidual explants were maintained in culture for up to 6 days. Attachment of the distal end of the villous tips to the decidua, their adherence to matrigel, and the appearance of EVTs breaking through from the tips were used as markers of viability and morphologic integrity as previously described by Genbacev et al (1993). Explants (from a single patient) were cultured in triplicate for each treatment point. Each experiment was repeated with at least six placentae and matched decidua. Decidua parietalis was also cultured alone as a control to assess that there was no trophoblast invasion prior to the establishment of the culture or degradation of blood vessels due to the culture conditions.

\section{Immunohistochemistry}

Placental-decidual co-cultures and decidual control tissues were fixed in the insert by immersion in cold 4\% paraformaldehyde for 1 hour and prepared for paraffin embedding. Histology was assessed on every 10th serial section using the standard hematoxylin eosin counterstaining protocol. For immunohistochemistry, 5- $\mu \mathrm{m}$ sections were deparaffinized in $x y-$ lene and rehydrated through a gradient series of ethanol in PBS. Endogenous peroxidase activity was blocked by incubation of the sections in 3\% hydrogen peroxide in methanol for 40 minutes. Antigens were unmasked using microwave pretreatment in $10 \mathrm{~mm}$ citrate buffer $\mathrm{pH} 6.0$, (sections were incubated for 10 minutes at high power in the DAKO pressure cooker; DAKO Diagnostics Canada Inc., Missisauga, Ontario, Canada) or $0.02 \%$ Triton X-100 (Sigma, St. Louis, Missouri) pretreatment for the Cdx-2 antibody. Immunostaining used the HRP-LSAB technique (LSAB kit; DAKO). (For antibodies used in the study, see Table 1.) Negative controls were carried out by omission of the primary antibody or incubation with nonimmune mouse lgG. Slides were developed in $0.075 \%$ (weight/ volume) 3,3-diaminobenzidine in PBS containing $0.002 \%$ (volume/volume) $\mathrm{H}_{2} \mathrm{O}_{2}$ with or without nickel 
Table 1. Antibodies Used in Study

\begin{tabular}{|c|c|c|c|}
\hline Antibody & Supplier & Dilution & Specificity \\
\hline Cytokeratin-7 & $\begin{array}{l}\text { Dako Diagnostics Canada Inc. (Missisauga, } \\
\text { Ontario, Canada) }\end{array}$ & $1: 300$ & Stains trophoblast and all epithelia \\
\hline$C d x-2$ & BioGenex (San Ramone, California) & $1: 50$ & $\begin{array}{l}\text { Stains intestinal epithelia and } \\
\text { trophoblast }\end{array}$ \\
\hline CD31 (PECAM-1) & Dako & $1: 50$ & Stains endothelial cells \\
\hline SHLA-G & Serotec (Oxford, United Kingdom) & $1: 100$ & $\begin{array}{l}\text { Stains extravillous trophoblast of cel } \\
\text { columns }\end{array}$ \\
\hline Smooth muscle actin & Dako & $1: 50$ & Stains smooth muscle cells \\
\hline
\end{tabular}

PECAM, platelet endothelial cell adhesion molecule; sHLA, soluble form of human leukocyte antigen.

enhancement (Vector Laboratories, Inc., Burlingame, California). After counterstaining with hematoxylin or nuclear fast red (Sigma), slides were dehydrated in an ascending ethanol series, cleared in xylene, and mounted with Permount (Fisher Scientific, Fair Lawn, New Jersey). Photomicrographs were taken using a Sony PowerHAD 3CCD color video camera DXC970MD (Sony of Canada Ltd., Willowdale, Ontario, Canada) and Northern Eclipse software.

\section{References}

Aplin JD (1991). Implantation, trophoblast differentiation and hemochorial placentation: Mechanistic evidence in vivo and in vitro. J Cell Sci 99:681-692.

Beck F, Erler T, Russell A, and James R (1995). Expression of Cdx-2 in the mouse embryo and placenta: Possible role in patterning of the extra-embryonic membranes. Dev Dyn 204:219-227.

Benirschke K and Kaufmann P. (1995). The pathology of the human placenta, 3rd ed. Benirschke $\mathrm{K}$ and Kaufmann $\mathrm{P}$, editors. London: Springer Verlag.

Boyd JD and Hamilton WJ (1970). The human placenta. Cambridge: Heffer and Sons.

Burton GJ, Jauniaux E, and Watson AL (1999). Maternal arterial connections to the placental intervillous space during the first trimester of human pregnancy: The Boyd collection revisited. Am J Obstet Gynecol 181:718-724.

Cartwright JE, Kenny LC, Dash PR, Crocker IP, Aplin JD, Baker PN, and Whitley GS (2002). Trophoblast invasion of spiral arteries: A novel in vitro model. Placenta 23:232-235.

Genbacev O, Jensen KD, Powlin SS, and Miller RK (1993). In vitro differentiation and ultrastructure of human extravillous trophoblast (EVT) cells. Placenta 14:463-475.

Genbacev O, Joslin R, Damsky CH, Polliotti BM, and Fisher SJ (1996). Hypoxia alters early gestation human cytotrophoblast differentiation/invasion in vitro and models the placental defects that occur in preeclampsia. J Clin Invest 97:540-550.

Hunt JS, Hsi BL, King CR, and Fishback JL (1991). Detection of class I MHC mRNA in subpopulations of first trimester cytotrophoblast cells by in situ hybridization. J Reprod Immunol 19:315-323.

Kadyrov M, Schmitz C, Black S, Kaufmann P, and Huppertz B (2003). Pre-eclampsia and maternal anaemia display reduced apoptosis and opposite invasive phenotypes of extravillous trophoblast. Placenta 24:540-548.
Kam EP, Gardner L, Loke YW, and King A (1999). The role of trophoblast in the physiological change in decidual spiral arteries. Hum Reprod 14:2131-2138.

Khong TY, De Wolf F, Robertson WB, and Brosens I (1986). Inadequate maternal vascular response to placentation in pregnancies complicated by pre-eclampsia and by small-forgestational-age infants. Br J Obstet Gynaecol 93:10491059.

Kovats S, Main EK, Librach C, Stubblebine M, Fisher SJ, and DeMars R (1990). A class I antigen, HLA-G, expressed in human trophoblasts. Science 248:220-223.

Le Bouteiller P (2000). HLA-G in the human placenta: Expression and potential functions. Biochem Soc Trans 28:208212.

Le Bouteiller P, Solier C, Proll J, Aguerre-Girr M, Fournel S, and Lenfant $F$ (1999). Placental HLA-G protein expression in vivo: Where and what for? Hum Reprod Update 5:223-233.

Lim KH, Zhou Y, Janatpour M, McMaster M, Bass K, Chun SH, and Fisher SJ (1997). Human cytotrophoblast differentiation/invasion is abnormal in pre-eclampsia. Am J Pathol 151:1809-1818.

Loke YW and King A (1995). Human Implantation. Cell Biology and Immunology. Cambridge: Cambridge University Press.

Lyall F (2002). The human placental bed revisited. Placenta 23:555-562.

Lyall F, Barber A, Myatt L, Bulmer JN, and Robson SC (2000). Hemeoxygenase expression in human placenta and placental bed implies a role in regulation of trophoblast invasion and placental function. FASEB J 14:208-219.

Lyall F, Bulmer JN, Duffie E, Cousins F, Theriault A, and Robson SC (2001). Human trophoblast invasion and spiral artery transformation: The role of PECAM-1 in normal pregnancy, preeclampsia, and fetal growth restriction. Am J Pathol 158:1713-1721.

Lyall F, Bulmer JN, Kelly H, Duffie E, and Robson SC (1999). Human trophoblast invasion and spiral artery transformation: The role of nitric oxide. Am J Pathol 154:1105-1114.

Pijnenborg R, Bland JM, Robertson WB, and Brosens I (1983). Uteroplacental arterial changes related to interstitial trophoblast migration in early human pregnancy. Placenta 4:397-413.

Pijnenborg R, Dixon G, Robertson WB, and Brosens I (1980). Trophoblastic invasion of human decidua from 8 to 18 weeks of pregnancy. Placenta 1:3-19. 
Pijnenborg R, Vercruysse L, Verbist L, and Van Assche FA (1998). Interaction of interstitial trophoblast with placental bed capillaries and venules of normotensive and preeclamptic pregnancies. Placenta 19:569-575.

Proll J, Blaschitz A, Hutter H, and Dohr G (1999). First trimester human endovascular trophoblast cells express both HLA-C and HLA-G. Am J Reprod Immunol 42:30-36.

Rodesch F, Simon P, Donner C, and Jauniaux E (1992). Oxygen measurements in endometrial and trophoblastic tissues during early pregnancy. Obstet Gynecol 80:283-285.

Rossant J and Cross JC (2001). Placental development: Lessons from mouse mutants. Nat Rev Genet 2:538-548.

Silberg DG, Swain GP, Suh ER, and Traber PG (2000). Cdx1 and cdx2 expression during intestinal development. Gastroenterol 119:961-971.

Thaler I, Manor D, Itskovitz J, Rottem S, Levit N, TimorTritsch I, and Brandes JM (1990). Changes in uterine blood flow during human pregnancy. Am J Obstet Gynecol 162: $121-125$.
Vicovac L, Jones CJ, and Aplin JD (1995). Trophoblast differentiation during formation of anchoring villi in a model of the early human placenta in vitro. Placenta 16:41-56.

Ziegler WF, Bernstein I, Badger G, Leavitt T, and Cerrero ML (1999). Regional hemodynamic adaptation during the menstrual cycle. Obstet Gynecol 94:695-699.

Zhou Y, Damsky CH, and Fisher SJ (1997a). Preeclampsia is associated with failure of human cytotrophoblasts to mimic a vascular adhesion phenotype. One cause of defective endovascular invasion in this syndrome? J Clin Invest 99:21522164

Zhou Y, Fisher SJ, Janatpour M, Genbacev O, Dejana E, Wheelock M, and Damsky CH (1997b). Human cytotrophoblasts adopt a vascular phenotype as they differentiate. A strategy for successful endovascular invasion? J Clin Invest 99:2139-2151. 